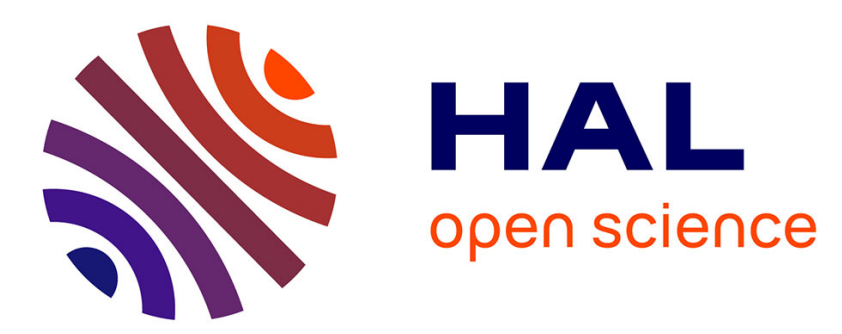

\title{
Static and Dynamic Light Scattering Near the Liquid Crystalline Blue Phase III - Isotropic Liquid Critical Point
}

Upindranath Singh, Peter Collings, Christopher Booth, John Goodby

\section{- To cite this version:}

Upindranath Singh, Peter Collings, Christopher Booth, John Goodby. Static and Dynamic Light Scattering Near the Liquid Crystalline Blue Phase III - Isotropic Liquid Critical Point. Journal de Physique II, 1997, 7 (11), pp.1683-1691. 10.1051/jp2:1997209 ． jpa-00248542

HAL Id: jpa-00248542

https://hal.science/jpa-00248542

Submitted on 1 Jan 1997

HAL is a multi-disciplinary open access archive for the deposit and dissemination of scientific research documents, whether they are published or not. The documents may come from teaching and research institutions in France or abroad, or from public or private research centers.
L'archive ouverte pluridisciplinaire HAL, est destinée au dépôt et à la diffusion de documents scientifiques de niveau recherche, publiés ou non, émanant des établissements d'enseignement et de recherche français ou étrangers, des laboratoires publics ou privés. 


\title{
Static and Dynamic Light Scattering Near the Liquid Crystalline Blue Phase III - Isotropic Liquid Critical Point
}

\author{
Upindranath Singh $\left({ }^{1}\right)$, Peter J. Collings $\left({ }^{2, *}\right)$, Christopher J. Booth $\left({ }^{3}\right)$ and \\ John W. Goodby $\left({ }^{3}\right)$
}

$\left({ }^{1}\right)$ Department of Physics, Mathematics, and Computer Science, University of the West Indies, Cave Hill, Bridgetown, Barbados

$\left(^{2}\right)$ Department of Physics \& Astronomy, Swarthmore College, Swarthmore, PA 19081, USA

$\left({ }^{3}\right)$ Department of Chemistry, University of Hull, Hull HU6 7RX, UK

(Received 22 May 1997, accepted 4 July 1997)

PACS.61.30.-v - Liquid crystals

PACS.64.70.Md - Transitions in liquid crystals

\begin{abstract}
Static and dynamic light scattering measurements, all using circularly polarized light, are used to investigate the third blue phase to isotropic liquid critical point in a mixture of S, S-4" (methylbutyl)phenyl-4' - (methylbutyl) biphenylcarboxylate (S,S-MBBPC) and its racemate. The intensity of light scattering and the relaxation time for the most strongly fluctuating chiral mode are found to be significantly greater than in a supercritical mixture of S,S-MBBPC. While the increase is substantial, there is no indication that any true divergence in these quantities is present. The results can qualitatively be explained by the simple phenomenological theory of Lubensky and Stark [3], which places this transition in the same universality class as the liquid-gas transition.
\end{abstract}

\section{Introduction}

Liquid crystal systems composed of chiral molecules or doped with chiral additives form the chiral nematic phase $\left(\mathrm{N}^{*}\right)$ instead of the nematic phase. In the chiral nematic phase, the direction of preferred orientation is not constant throughout the sample, but rotates in helical fashion. The distance over which the direction of preferred orientation rotates through $2 \pi$ radians is called the pitch of the chiral nematic phase, which can be as small as $100 \mathrm{~nm}$ or larger than the size of the sample. If the pitch is greater than about $500 \mathrm{~nm}$, the chiral nematic phase undergoes a phase transition directly to the isotropic liquid phase (ISO) as the temperature is increased. In systems with a smaller pitch, however, the transition to the isotropic phase is via one, two, or three blue phases. These blue phases are characterized by a local order different from that of the chiral nematic phase, but more interesting is the fact that a collection of defect lines or disclinations spontaneously forms. In two of the blue phases $\left(\mathrm{BP}_{\mathrm{I}}\right.$ and $\left.\mathrm{BP}_{\mathrm{II}}\right)$. these disclination lines form body-centered cubic and simple cubic lattices, respectively. In the blue phase that forms at the highest temperature in the most chiral systems

$\left({ }^{*}\right)$ Author for correspondence (e-mail: pcollin1@swarthmore.edu) 
$\left(\mathrm{BP}_{\mathrm{III}}\right)$, the disclination lines (if they are present at all) form an amorphous structure. See reference [1] for several review articles on the blue phases.

Recent experimental work has shown that the phase transition line between this amorphous blue phase and the isotropic liquid phase ends at a critical point [2]. The evidence for this comes from optical activity and specific heat experiments. By varying the concentration of the stereoisomers to produce mixtures of decreasing pitch, evidence for a discontinuity in the optical activity gradually disappears. Measurements of the specific heat also demonstrate that the two phase region disappears, with one phase converting to the other continuously. The measured exponents are mean-field, perhaps due to the fact that the critical region may be experimentally inaccessible. A phenomenological theory that introduces a new order parameter finds that the $\mathrm{BP}_{\text {III }}$-ISO transition is in the same universality class as the liquid-gas transition [3].

Although static and dynamic light scattering has been performed on one of the systems exhibiting a critical point, it was done far above the critical point where $\mathrm{BP}_{\mathrm{III}}$ converts to the isotropic phase without a phase transition [4]. It therefore displayed no true divergence and no critical slowing down, but simply a gradual change from a temperature dependence typical of $\mathrm{BP}_{\text {III }}$ to one typical of the isotropic phase. The change in temperature dependence coincided with the region in which the specific heat showed a broad anomaly. Also, there has been a static light scattering measurement done on a compound that may be close to the critical point since its measured latent heat is extremely small [5], but these measurements also showed no true divergence in the light scattering intensity at the transition [6]. Obviously of more interest are static and dynamic light scattering measurements on a near-critical mixture. How large are the fluctuations at the critical point as revealed by light scattering? How do they compare with the fluctuations in the supercritical region? Is there appreciable slowing down of the fluctuations near the critical point? These questions are interesting, since unlike the $\mathrm{N}^{*}$-ISO transition, the order parameter appropriate to the $\mathrm{BP}_{\mathrm{III}}-\mathrm{ISO}$ transition does not describe simple orientational order, which can be probed directly through light scattering. Still, any strong coupling between the $\mathrm{BP}_{\mathrm{III}}-\mathrm{ISO}$ order parameter and the orientational order parameter should reveal itself in the light scattering data.

The results of light scattering measurements on a near-critical mixture of S,S-, R,R-, S,R-, and R.S-4" -(methylbutyl)phenyl- $4^{\prime}-$ (methylbutyl)biphenylcarboxylate (MBBPC) are reported in this paper. The intensity of the fluctuations and the degree of slowing down in this near-critical mixture are quite enhanced over the supercritical mixture, but no true divergent behavior is evident. This indicates that the fluctuations being probed by the light scattering measurements, even though they are chiral in form, are not strongly coupled to the order parameter responsible for the critical behavior.

\section{Theory}

Perhaps the most important component of the phenomenological theory is the realization that the spatially averaged nematic alignment tensor describing the orientational order, $\langle\mathbf{Q}(\mathbf{r})\rangle$, is zero in both $\mathrm{BP}_{\mathrm{III}}$ and the isotropic phase [3]. However, a new scalar order parameter, $\langle\psi(\mathbf{r})\rangle=\langle(\nabla \times \mathbf{Q}(\mathbf{r})) \cdot \mathbf{Q}(\mathbf{r})\rangle$. is not zero in these phases. If a coarse-grained Hamiltonian is formed by averaging over wavevectors greater than some value, and if the normal terms for a chiral system, the normal liquid-gas terms, and the lowest order coupling term from the coarse-graining procedure are retained, then a free energy density identical to the liquid-gas free energy density results,

$$
f=\frac{1}{2} a_{0} r\langle\phi\rangle^{2}+\frac{1}{4} u_{0}\langle\phi\rangle^{4}-h\langle\phi\rangle
$$




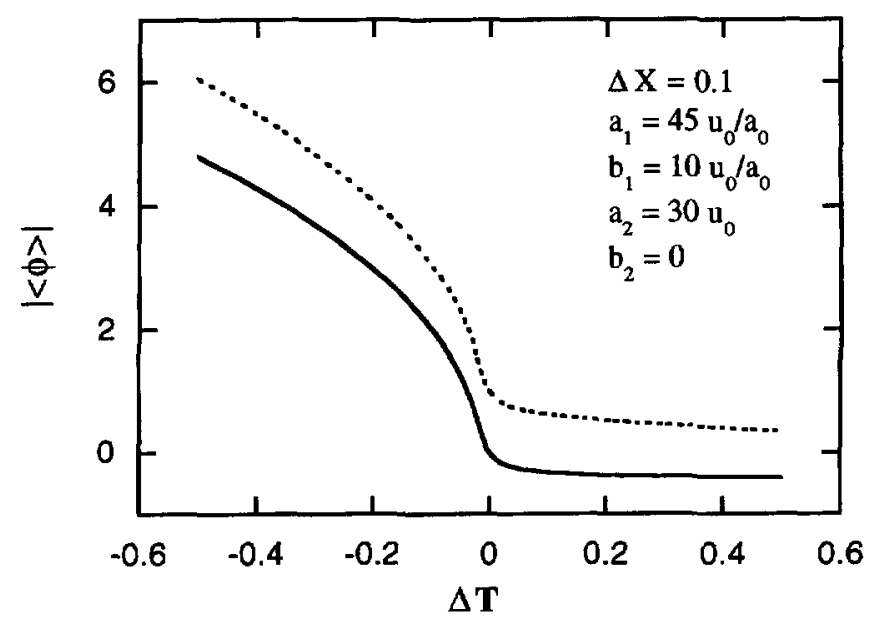

Fig. 1. - Order parameter versus temperature according to the phenomenological theory of Lubensky and Stark. Solid line: order parameter; dashed line: order parameter with a linear background added.

The theoretical temperature and its conjugate field are $r$ and $h$, respectively, $\langle\phi\rangle$ is the difference between $\langle\psi\rangle$ and its value at the critical point, $\langle\psi\rangle_{\mathrm{C}}$, and $a_{0}$ and $u_{0}$ are constants $(r=h=0$ at the critical point). Experimentally, the temperature $T$ and chiral fraction $X$ are accessible, so these are assumed to be related linearly to $r$ and $h$,

$$
\begin{aligned}
& r=a_{1} \Delta T+b_{1} \Delta X \\
& h=a_{2} \Delta T+b_{2} \Delta X,
\end{aligned}
$$

where $\Delta T=T-T_{\mathrm{C}}$ and $\Delta X=X-X_{\mathrm{C}}\left(T_{\mathrm{C}}\right.$ and $X_{\mathrm{C}}$ are the temperature and chiral fraction at the critical point). Finding values for $\langle\phi\rangle$ at particular values of $\Delta T$ and $\Delta X$ requires that a cubic equation be solved. Proper choice of the values of $a_{1}, a_{2}, b_{1}$, and $b_{2}$ (in terms of $u_{0}$ and $u_{0} / a_{0}$ ) produces curves which closely follow the optical activity of the near-critical mixture. Such a curve is shown in Figure 1, where constants that closely describe the optical activity of this mixture have been used [2]. Since a linear background is necessary in order to get good agreement between theory and the optical activity data, order parameter curves with and without such a background are shown in Figure 1. In addition, the specific heat can be derived from the theoretical free energy, and it too qualitatively describes the specific heat data of this near-critical mixture.

As has been pointed out, slowly varying background terms must be added in order to properly fit the optical activity and specific heat experimental data. These terms could very well be related to the build-up of true long-range orientational order in the chiral nematic phase, as opposed to the establishment of a more local type of order in the $\mathrm{BP}_{\mathrm{III}}$ and isotropic phases.

\section{Experimental Method}

The light scattering experiment was similar to the one done previously on supercritical S,S-MBBPC [4], but this time the sample was a mixture of $45 \% \mathrm{~S}, \mathrm{~S}-\mathrm{MBBPC}$ and $55 \%$ racemic MBBPC (equal concentrations of S,S-, R,R-, S,R-, and R,S-MBBPC). Previous specific heat and optical activity measurements demonstrated that this mixture is very close to the critical mixture [2]. The sample was contained in a $1 \mathrm{~cm}$ square spectrophotometer cell, placed in an 
aluminum oven. Small holes in the oven allowed light to enter and leave along two perpendicular paths. The temperature of the oven was controlled by an Instec $\mathrm{mK} 1$ controller, and in the temperature range of this experiment it regulated the oven temperature to within a few $\mathrm{mK}$. Static and dynamic light scattering measurements were performed using $488 \mathrm{~nm}$ light from a $10 \mathrm{~mW}$ argon ion laser and a $90^{\circ}$ scattering angle for a temperature interval that included $\mathrm{BP}_{\mathrm{I}}, \mathrm{BP}_{\mathrm{III}}$, and the isotropic phases. Measurements were taken (1) with only Right Circularly Polarized (RCP) light both incident on the sample and detected by the photomultiplier tubes, and (2) with Left Circular Polarized (LCP) light both incident on the sample and detected by the photomultiplier tubes. The first arrangement is sensitive to the most strongly fluctuating chiral mode in this sample and the second arrangement is sensitive to the most weakly fluctuating chiral mode in this sample [7]. In both arrangements, a nonchiral fluctuating mode contributes to the detected light intensity at the $1 \%$ level and another chiral fluctuating mode contributes at less than the $0.1 \%$ level. A cross correlation technique using a beam splitter and two photomultiplier tubes was used to eliminate the effects of afterpulsing in the photomultiplier tubes, since these afterpulses were delayed by a fraction of a microsecond and therefore interfered with the photon correlation due to fluctuations, which were also in the microsecond range. The correlator was a Malvern $7032 \mathrm{ES}$ using a $50 \mathrm{~ns}$ sample time. All data were taken on heating, starting in $\mathrm{BP}_{\mathrm{I}}$ and stopping significantly above the $\mathrm{BP}_{\mathrm{III}}$-ISO transition. Exponential fits to the correlation function at each temperature allowed the relaxation time to be measured.

Due to the fact that the amount of light scattered by the sample in the vicinity of the transition and in the blue phases was much higher than expected, an additional turbidity experiment was performed. The same sample, oven, optical components, and light source were used, but the intensity of light incident on the sample was measured by splitting off a small percentage of the incident light and measuring it with a semiconductor detector. In addition, the intensity of light emerging from the sample was measured by a similar semiconductor detector placed behind a small aperture. The aperture prevented light scattered at all but the smallest of angles from reaching the detector. This arrangement ensured that multiple scattering did not affect the turbidity measurements. Measurements were taken in heating with both right and left circularly polarized light incident on the sample. Rather than calibrate the beam splitter in front of the sample, all ratios of the intensity emerging from the sample to the intensity split off from the incident beam were divided by the same ratio measured at a temperature more than $10 \mathrm{~K}$ above the transition. The relative turbidity of the sample is then the negative of the natural logarithm of this quotient of ratios.

\section{Experimental Results}

The intensity of light scattering as a function of temperature is shown in Figure 2. At high temperatures, the behavior is similar to measurements made in the supercritical region of this system, with the RCP/RCP arrangement showing slightly stronger scattering than the LCP/LCP arrangement. At lower temperatures (near the transition and in the blue phases), the intensity of light scattering with the RCP/RCP arrangement decreases, even to the point that it is significantly lower than with the LCP/LCP arrangement. Nothing like this was seen in the supercritical region of this same system [4] or in back-scattering measurements using $1 \mathrm{~mm}$ samples for mixtures of $\mathrm{S}, \mathrm{S},-\mathrm{MBBPC}$ with a nonchiral liquid crystal [8]. This suggests that this critical mixture scatters light to such a degree that multiple scattering effects in this fairly large sample decrease the amount of light reaching the photomultiplier tubes.

The relaxation times measured with both polarization arrangements during the same experimental run are presented in Figure 3. Again, at high temperatures the data are similar to 


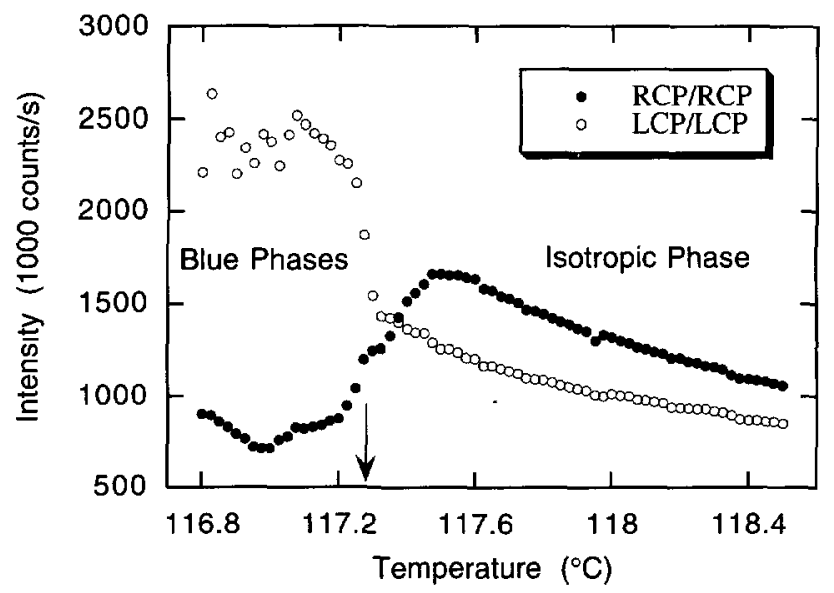

Fig. 2. - Light scattering intensity as a function of temperature. $\bullet$ : right circularly polarized incident light with detection of only right circularly polarized light; $\circ$ : left circularly polarized incident light with detection of only left circularly polarized light. The wavelength is $488 \mathrm{~nm}$. The arrow indicates the most likely location of the $\mathrm{BP}_{\mathrm{III}}$-ISO transition based on the LCP/LCP data.

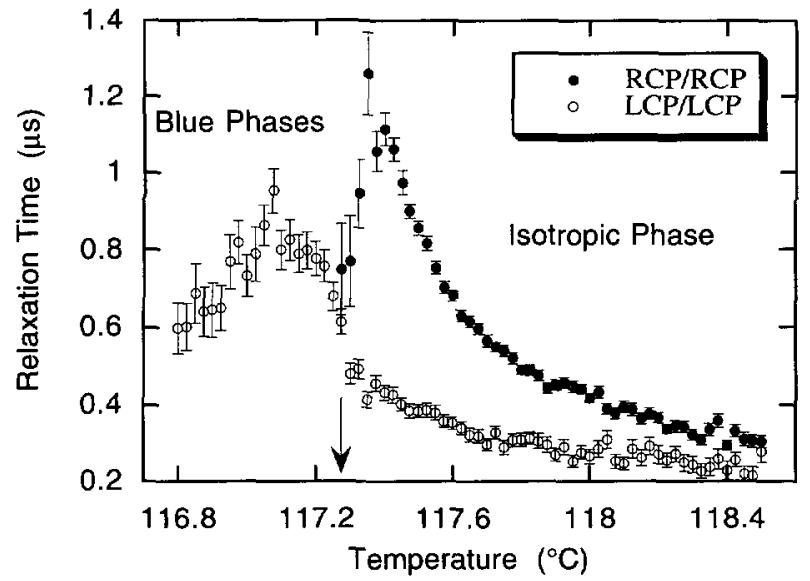

Fig. 3. - Relaxation time as a function of temperature. $\bullet$ : right circularly polarized incident light with detection of only right circularly polarized light; o: left circularly polarized incident light with detection of only left circularly polarized light. The wavelength is $488 \mathrm{~nm}$. The arrow indicates the most likely location of the $\mathrm{BP}_{\text {III }}$-ISO transition based on the LCP/LCP data.

data taken in the isotropic phase of other highly chiral systems. Note, however, that for about the same range of temperature just above the $\mathrm{BP}_{\mathrm{III}}-\mathrm{ISO}$ transition for which the RCP/RCP arrangement shows reduced light scattering intensity, the relaxation time shows significantly larger error estimates and in some cases reduced values. The relaxation time error estimates for the RCP/RCP arrangement are significantly larger in the blue phases due to a loss of photon correlation in the scattered light, so these data are not shown. This also suggests that the sample is so highly scattering in the blue phases and part of the isotropic phase for RCP light that multiple scattering effects make correlation measurements difficult. 


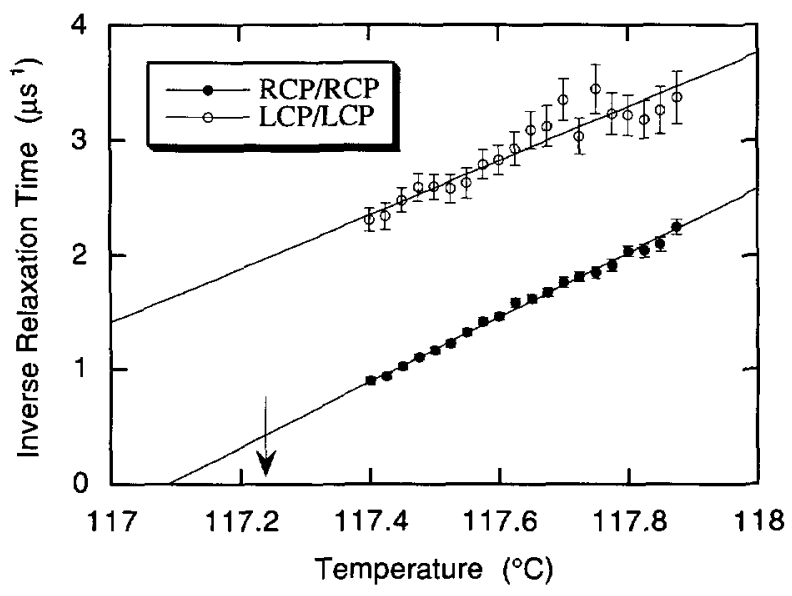

Fig. 4. - Inverse relaxation time as a function of temperature in the isotropic phase close to the transition. The arrow indicates the most likely location of the BP III $^{-I S O}$ transition based on the data shown in Figures 2 and 3. The straight lines are weighted linear least square fits to the data.

The relaxation times measured for this near-critical mixture are also longer than measured using this same chiral compound but away from the critical region. For example, the longest relaxation time measured near the transition in the near-critical mixture is over $1 \mu \mathrm{s}$, whereas it is less than $0.5 \mu \mathrm{s}$ in the conversion region of the supercritical sample [4].

The inverse of the relaxation time is proportional to the linewidth of the scattered light, and according to mean-field theory for the $\mathrm{N}^{*}$-ISO transition should be linearly proportional to temperature in the isotropic phase away from the critical point $[9,10]$. Figure 4 shows the inverse relaxation time as a function of temperature for a small region in the isotropic phase near the transition but far enough away so that multiple scattering effects should not affect the measurements. A weighted linear least square fit to the $\mathrm{RCP} / \mathrm{RCP}$ data extrapolates to a true divergent temperature, $T^{*}(q), 0.15 \mathrm{~K}$ below the transition temperature, $T_{\mathrm{C}}$, providing quantitative evidence that the relaxation time significantly increases in this near-critical mixture but does not truly diverge. This difference between the transition temperature and the extrapolated temperature (about $0.15 \mathrm{~K}$ ) is extremely similar to the result found using light scattering intensity measurements in a mixture of $40 \mathrm{wt} \% \mathrm{~S}, \mathrm{~S}-\mathrm{MBBPC}$ in a nematic host [8], and much less than this temperature difference in supercritical S,S-MBBPC using relaxation time measurements (about $0.55 \mathrm{~K}$ ) [4].

To test whether multiple scattering is causing these effects, a measurement of the relative turbidity was made for both RCP and LCP incident light. The results are shown in Figure 5. Since the turbidity is a measure of the amount of light scattered in all directions, the data substantiate just how much stronger the scattering of RCP light is compared to LCP light in the region of the transition and blue phases. For example, just below the transition, the turbidity for RCP light is 3.5 times that for LCP light. That translates to a intensity of scattered light that is over 30 times greater. This should be compared to a maximum of only a factor of 2 for the intensity of scattered RCP light versus LCP light in the supercritical mixture [4], but one might now wonder if multiple scattering effects might have slightly decreased the intensity of RCP light detected in this experiment. Even the backscattering measurements revealed a factor of only about 3 in the ratio of scattered light intensities near the transition [8]. These turbidity measurements therefore indicate that the amount of light scattered for the strongest fluctuating chiral mode is significantly larger than in the other chiral systems studied to date. 


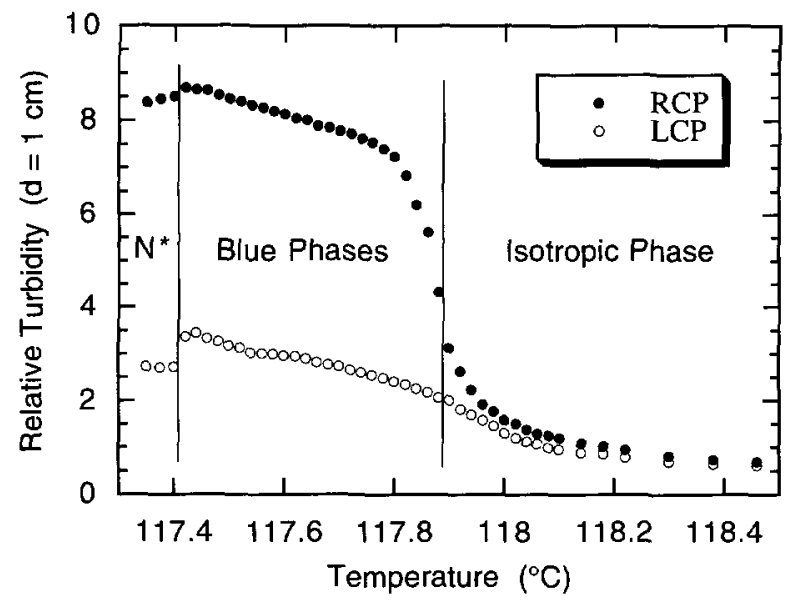

Fig. 5. - Turbidity as a function of temperature. •: right circular polarized light; o: left circularly polarized light. The wavelength is $488 \mathrm{~nm}$. The vertical lines indicate the most likely locations for the $\mathrm{N}^{*}-\mathrm{BP}_{1}$ and $\mathrm{BP}_{11}-\mathrm{ISO}$ transitions.

It should be pointed out that there is a difference of about $0.6 \mathrm{~K}$ between the BP III $_{-}$ISO transition temperature as measured in the scattering and turbidity measurements. Although the same sample and oven were used in both experiments, the scattering measurements were made after the oven had been near the transition temperature for almost a day, while the turbidity experiments were made after the oven had been heated to near the transition temperature for about an hour. In addition, the turbidity measurements took much less time to acquire than the light scattering measurements. Since the sample was quite large (about $1 \mathrm{~cm}^{3}$ ) and the temperature of the surrounding metal oven was actually what was controlled, the temperature of the sample might have been closer to the oven temperature during the scattering experiment than during the turbidity experiment. Since both experiments were done on heating, this would account for the slightly lower measured transition temperature in the scattering experiment.

\section{Discussion}

Since the pitch of the near-critical mixture is about $230 \mathrm{~nm}$ while the pitch of the supercritical mixture is about $100 \mathrm{~nm}$ [11], one must first ask if the increased scattering intensity and longer relaxation time might simply be due to a change in pitch. In the isotropic phase, mean field theory for the $\mathrm{N}^{*}$-ISO transition indicates that both the scattering intensity and relaxation time are inversely proportional to $\left[A(T)-L q\left(2 q_{0}-q\right)\right]$, where $A(T)$ is the coefficient of the term quadratic in the order parameter in the free energy, and $L$ is the coefficient of the term quadratic in the gradient of the order parameter (intensity) or the coefficient of a dissipation term (relaxation time) $[9,12] . \quad q_{0}$ is equal to $4 \pi$ divided by the pitch, and the scattering wavevector in the sample $q$ is equal to $\left(4 \pi n / \lambda_{0}\right) \sin (\theta / 2)$, where $n$ is the average index of refraction of the sample, $\lambda_{0}$ is the wavelength of the light in a vacuum, and $\theta$ is the scattering angle. Since $q_{0}>q$, the lower value of $q_{0}$ for the near-critical mixture would suggest smaller values for the scattering intensity and relaxation time if $A(T)$ and $L$ do not change. The fact that just the opposite is true means that the coefficients of the free energy are changing, as one would expect near a critical point. Another way of illustrating this same point is to realize that 
the difference between the transition temperature and the extrapolated divergent temperature is given by mean field theory for the $\mathrm{N}^{*}$-ISO transition as

$$
T_{\mathrm{C}}-T^{*}(q)=\left(T_{\mathrm{C}}-T^{*}\right)-\frac{L q}{A_{0}}\left(2 q_{0}-q\right)
$$

where $T^{*}$ is the temperature at which $A(T)$ is zero, i.e., $A(T)=A_{0}\left(T-T^{*}\right)$. Decreasing $q_{0}$ normally would increase $T_{\mathrm{C}}-T^{*}(q)$ if the other parameters remain constant, whereas as has been pointed out before, $T_{\mathrm{C}}-T^{*}(q)$ is 3-4 times smaller for the near critical mixture as compared to the supercritical mixture. Again, other parameters describing the transition must be changing significantly.

Clearly the near-critical mixture shows some unusual light scattering phenomena when compared to similar systems far from the critical point. This lends additional experimental evidence for the existence of the critical point in this system near a chiral fraction of $45 \%$. Of more importance, however, is that fact that although the order parameter appropriate for this transition must be coupled to the orientational order parameter to some extent, this coupling is obviously weak enough that no true divergent behavior in the light scattering is observed. This is reasonable given that $\langle\phi\rangle$ depends on the spatial variation of the nematic alignment tensor, rather than the nematic alignment tensor itself. Perhaps $\langle\phi\rangle$ is proportional to the amplitude of the fluctuations of the orientational order parameter, in which case a light scattering experiment would be sensitive only to weakly diverging higher order fluctuations. Only experiments done extremely close to the $\mathrm{BP}_{\mathrm{III}}$-ISO transition would then have a chance of revealing true divergent behavior.

It is interesting to note how well the phenomenological theory can account for these measurements. If the simplifying assumption is made that the total amount of light scattering and therefore the turbidity is linearly dependent on $\langle\phi\rangle$, then the curves of Figure 1 should resemble the turbidity data. This is qualitatively true, especially for the curve with the linear background added. As mentioned previously, this background contribution is probably related to the build-up of long-range nematic type ordering of the chiral nematic phase, which is interrupted by the more local order of the blue phases. It should also be pointed out that the turbidity data of Figure 5 are very similar to previous light scattering measurements in a backscattering configuration taken on a compound that is close to the critical point but still has a measurable latent heat [6].

\section{Conclusion}

These results are preliminary, since measurements of the light scattering intensity and relaxation times throughout the $\mathrm{BP}_{\mathrm{III}}$-ISO phase transition region must be performed in a way free from the effects of multiple scattering. These will require a smaller sample and result in a loss of scattering signal. Preparations for such measurements are being made at this time. Still, the light scattering and turbidity measurements reported here are revealing. The intensity of light scattering and the relaxation times are significantly increased near the BP III-ISO critical point, but with no indication of true divergent behavior. In addition, the general nature of these results can be explained by a simple phenomenological theory employing a new scalar order parameter. 


\section{Acknowledgments}

These results are based on work supported by the National Science Foundation under Grant No. DMR-9224617. One of the authors (U.S.) wishes to acknowledge support from the UWI/IDB Development Fund. Useful discussions with M.A. Anisimov, P.H. Keyes, T.C. Lubensky, and H. Stark are gratefully acknowledged.

\section{References}

[1] Seideman T., Rep. Prog. Phys. 53 (1990) 659; Wright D.C. and Mermin N.D., Rev. Mod. Phys. 61 (1989) 385; Crooker P.P., Liq. Cryst. 5 (1989) 751; Hornreich R.M. and Shtrikman S., Mol. Cryst. Lıq. Cryst. 165 (1989) 183; Belyakov V.A. and Dmitrienko V.E., Usp. Fiz. Nauk 146 (1985) 369 [Sov. Phys. Usp. 28 (1985) 535].

[2] Kutnjak Z., Garland C. W., Schatz C.G., Collings P.J., Booth C.J. and Goodby J.W. Phys. Rev. E 53 (1996) 4955.

[3] Lubensky T.C. and Stark H., Phys. Rev. E 53 (1996) 714.

[4] Kutnjak Z., Garland C.W., Passmore J.L. and Collings P.J., Phys. Rev. Lett. 74 (1995) 4859.

[5] Voets G., Ph.D. Thesis, University of Leuven, Belgium, 1992 (unpublished).

[6] Becker J.B. and Collings P.J., Mol. Cryst. Liq. Cryst. 265 (1995) 163.

[7] For a recent review, see Collings P.J., Mod. Phys. Lett. B 6 (1992) 425.

[8] Wyse J.E. and Collings P.J., Phys. Rev. A 45 (1992) 2449.

[9] Zink H. and Van Dael W., Liq. Cryst. 5 (1989) 899.

[10] Singh U. and Keyes P.H., in reference [7].

[11] Yang D.K. and Crooker P.P., Phys. Rev. A 35 (1987) 4419.

[12] Koistinen E.P. and Keyes P.H., Phys. Rev. Lett. 74 (1995) 4460. 